\title{
A Flexible Display by Integrating a Wall-Size Display and Steerable Projectors
}

\author{
Li-Wei Chan ${ }^{1}$, Wei-Shian Ye ${ }^{2}$, Shou-Chun Liao ${ }^{2}$, Yu-Pao Tsai ${ }^{3,4}$, \\ Jane $\mathrm{Hsu}^{1,2}$, and Yi-Ping Hung ${ }^{1,2,3}$ \\ ${ }^{1}$ Graduate Institute of Networking and Multimedia, \\ National Taiwan University, Taipei, Taiwan \\ ${ }^{2}$ Dept. of Computer Science and Information Engineering, \\ National Taiwan University, Taipei, Taiwan \\ ${ }^{3}$ Institute of Information Science, Academia Sinica, Taipei, Taiwan \\ ${ }^{4}$ Dept. of Computer and Information Science, \\ National Chiao Tung University, Hsinchu, Taiwan \\ \{hung, yjhsu\}@csie.ntu.edu.tw
}

\begin{abstract}
Many wall-size display systems are built to provide large-scale visualization. These systems may be quite successful for some limited applications, but are very inflexible, since these systems only have fixed display regions. This paper integrates steerable projectors whose beam can be moved under computer control onto a wall-size display system to strengthen its display ability. With the steerable projectors, the integrated display system, named Flexible Display, provide an extendable display region. This consists of a large-scale display region and several movable display regions, such that the integrated display system has great potential in the area of human-computer interaction and information visualization. This paper applies the Flexible Display to a virtual museum application to give the users fluent navigation experience. For the application, the Flexible Display provides the following functions: 1) intensity and resolution enhancement of sub-region of display wall, 2) information augmentation, and 3) "stepping user interfaces" for its viewers interacting with display wall.
\end{abstract}

\section{Introduction}

Large displays, with the large-scale, high-resolution displaying capability, are highly applicable in many applications and public places. For example, information visualization in transport centers such as in airports and train stations; advertisement, bargaining, and information retrieval of purchasing in shopping center or markets; virtual reality, visual effect demonstration of artifacts or environments in museum or in an exhibition room. With its applicability and being increasingly found in public places, large displays have shown its importance as a ubiquitous technology in our future life.

Although benefits from embedding a large display in the environment are attractive, building such a display system is not easy. Numerous of attempts have been made by scholars to show how tiled projectors, forming projector arrays, to provide single large display. However, this kind of projector system is fixed and always requiring time-consuming calibration process. Besides, occupying large floor space also 
makes it impractical in a normal environment setting. Raskar et al. [5] proposed that an ad-hoc cluster of projectors can create a self-configuring display on even a nonplanar wall. Their approach has greatly reduced the process of calibration. Claudio Pinhanez $[6,7]$ makes projector-enabled displays a big step toward ubiquity by introducing steerable projectors into the environment. The steerable projector is composed of a projector and a mirror mounted on the pan-tile unit. Rotating the mirror can freely direct the beams onto almost everywhere in the environment. In summary, a fixed wall-size display is capable of presenting large scale and high resolution imagery, while a steerable projector is charactering on its ubiquity. Determining which projector system to be deployed depends on the application's needs.

In this paper, we are investigating how a wall-size display and steerable projectors coexisted in the environment can gain benefits from each other. We also show that how our system enriches interactions between the display wall and the viewers by leveraging strengths of fixed and steerable projector settings. The system, we called Flexible Display, offers advantages towering previous wall-size display systems on both its presentation and interaction abilities. We propose following functions of our system: 1) intensity and resolution enhancement of sub-region of display wall, 2) information augmentation, and 3) stepping user interfaces interacting with display wall. The details of these functions are described in section 2 . We also demonstrated the system in a museum application.

This research investigates the impacts and its applicability of joining both fixed and steerable projectors in the environment. This paper starts by describing several systems offering projective display to environment and their limitations. We then propose that the Flexible Display, leveraging strengths from the fixed and steerable projector settings, transcends previous systems on both its presentation and interaction abilities. Section 2 details the scenario and functions provided by the Flexible Display. Section 3 describes the core techniques required of the system. Section 4 presents a museum application, followed by the conclusion and future work in Section 5.

\section{Scenarios and Functions}

Our system (Flexible Display) can be extensively used in variety of applications in public places. Here is a sample scenario in a museum: Visiting to museum, Susan enters the intelligent exhibition room, where a projective wall-size display is on a particular wall. Knowing Susan's entrance, the display wall shows a question asking whether it is Susan's first time to attend the exhibition. At the same time, the steerable projector moves its projection, showing two buttons, labeled "Y/N", on the floor nearby Susan's foots. Susan steps on the "Y" button. The display then starts a movie clip introducing several very exhibits of the exhibition. The steerable projection, in the meantime, highlights the sub-regions of display wall to give guidance to the viewers' focus. Following the steerable projection, Susan finds it is an easier way to perceive the information delivered by the clip.

While the movie ends, the steerable projector displays several icons of artifacts on the floor. Susan steps on one of them. The following is the display wall shows the selected artifact and the steerable projector augments some description over the display wall. After that, she moves the steerable projection by dragging a stylus on her PDA. She then can appreciate every detail of that artifact. 


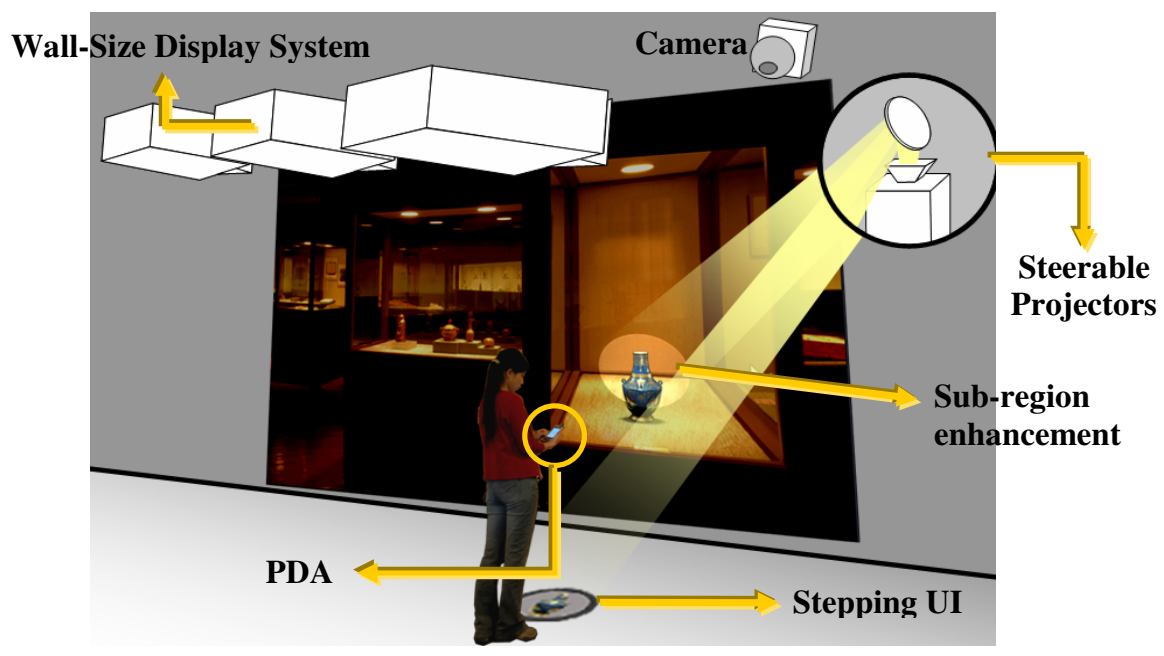

Fig. 1. A Diagram Depicting the Museum Scenario

The Flexible Display consists of following components:

1. A few projectors fixed on the ceiling, cooperatively projecting large scale display on a particular wall. In the implementation, we use three projectors to form a wallsize display system.

2. At least one steerable projector, offering moveable projection for polishing portion of display wall, augmenting information over the display or the environment, and delivering projective user interfaces which are easier accessed by the viewers.

3. A camera mounting on upper-side of display wall, for detecting the viewers' interaction with the projective user interface.

4. PDAs, that the viewers can move the steerable projection by dragging a stylus, detailing a part of the display.

We can now summarize three functions provided by our system.

1) intensity and resolution enhancement of sub-region of display wall,

2) information augmentation,

3) stepping user interface interacting with display wall,

\subsection{Intensity and Resolution Enhancement of Sub-region of Display Wall}

Current researches aim to create large, high-resolution displays by presenting improved methods of creating a "projector mosaic" [4] which is a collection of projected images combined to form one large display. These approaches usually uses numerous of projectors, causing high cost and troublesome calibration. In our previous work [8], we proposed a multi-resolution approach in the sense that the audience only focuses on a part of the projected area, so only this area requires higher resolution. Therefore, the multi-resolution approach requires only a few projectors, reducing the cost. In this approach, we assume the user always looks at the center of the display, where we 
called Fovea Region, is to be displayed in high resolution. The other region, called Peripheral Region, only provides the user an overview of the displayed content, and thus only requires lower resolution. Therefore only two projectors, each serving for one region, are used to build a two-level display.

Based on the previous approach, we further replace the projector served for Fovea Region with steerable projectors. Since the steerable projectors can freely move their projections, our system can detail every part of the display where the high resolution is required. To create large scale high resolution coverage, we combine only a few conventional projectors and at least one steerable projector. The conventional projectors which fixed somewhere form a projector cluster are to provide large coverage projective display on the wall, while the steerable ones are to provide smaller but higher resolution projections. Before the fixed and steerable projectors cooperatively projecting one seamless display, we need to apply calibrations both on the wall-size display system and the steerable projectors. We leave the technique details in section 3. Compared to previous projector-based large display, our system is low cost for its large scale high resolution display enacted by only a few projectors.

\subsection{Information Augmentation}

The wall-size display system and the steerable projectors though are to display information on the surface, in our system, they serve with different purposes. Being partners of the wall-size display system, the steerable projectors are not only to polish portions of the display, but can also be used to augment additional information of the content. For example, while the large display presents artifacts, the steerable projections can augment related information around the artifacts. Since the steerable projectors have higher intensity, their projection can help the viewers easily perceive the information. Besides, with the mobility of the steerable projections, the steerable projectors can extend the display wall by projecting augmentations outside the coverage of the display wall, making the displaying more flexible. Figure 7 shows that an artifact and its description are displayed on the wall. In this figure, the description occupies space too large to be contained in the display wall. The steerable projection extends the display wall to show the full description.

\subsection{Stepping User Interface Interacting with Display Wall}

As we show in the scenario, the display wall and the steerable projections work together to provide stepping interfaces to the viewers. So that Susan could answer a question from the display wall by simply moving her foot. To provide everywhere stepping user interface, a steerable projector coupled with a camera can create an active region around the viewers. The viewers interact with the large display by stepping on widgets within the active region. Claudio Pinhanez [6] had demonstrated that the steerable projection to the environment can be versatile, creating ubiquitous interfaces for its users interacting with the environment. In this case, we show such interactions can be applied to offer direct and intuitive interactions between the display wall and the viewers. 


\section{System Design}

The Flexible Display consists of a wall-size display system, steerable projectors, cameras, and personal handhelds. We may divide our system in three components: calibration of wall-size display system, calibration of steerable projectors regarding to the wall-size display system, i.e., the display wall, and the environment, and the interaction approaches. In the following, we describe each component and the required techniques.

\subsection{Calibration of the Wall-Size Display System}

While creating one large multi-projector display, we primarily have to solve two problems: geometric misalignment and photometric variations.

For the geometric problem, in the past, most relative research uses a fixed-lens camera to do geometric calibration [1,2]. However, their method requires that the entire projected area has to be completely visible by the fixed-lens cameras. The calibration may further degrade the accuracy of the measurements when the projected area becomes large. In order to increase calibration accuracy, here we utilized a technique similar to the technique adopted by Chen et al. [3] to increase the measurement resolution by combining several zoom-in images acquired by a pan-tilt-zoom camera. In addition, the zoom-in camera views have lens distortion that will increase the calibration error so lens correction is needed for all the zoom-in images.

For the photometric variation problems, readers can refer to the survey paper [11], where color variation in multi-projector displays has been classified clearly. Moreover, the projectors used to build a multi-projector display are generally video projectors because video projectors can provide more-accurate color representation. Therefore all projectors are considered to be video projectors here.

When we create the multi-projector display, there are obvious seams caused by the different chromatic response among projectors. For example, the display shows severe color differences when simply displaying pure red, green or blue due to the non-identical color gamut of each projector. Therefore in order to guarantee that any input-RGB color looks exactly the same over the display region, the common color gamut of the display should be determined. In addition, the non-linear behavior of the display and projectors also has to be considered and all input-RGB values have to be adjusted accordingly.

Another photometric problem is the non-uniformity of luminance over the display region. For example, the luminance on overlapped region is very noticeable obvious and luminance variation spatially inside a projector itself can also distract an audience from watching the display. Thus, in order to seamlessly merge the images without any obvious luminance non-uniformity, a luminance adjustment technique similar to [10] is used to smoothly combine the images into one large picture.

Therefore with regard to the two photometric variation problems described above, we utilized a two-phase photometric calibration method to not only solve chrominance variation problem among projectors but also to reduce perceptually the luminance variation over the tiled display. Figure 2 shows an image projected by the wall-size display system after geometric and photometric calibrations. More details can be found in our previous work [12]. 


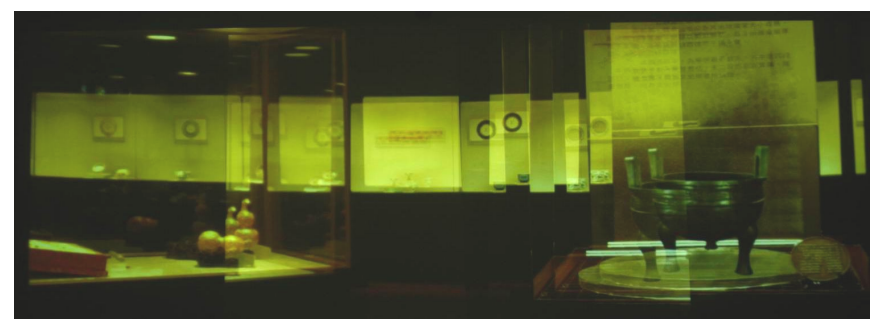

(a) without calibration

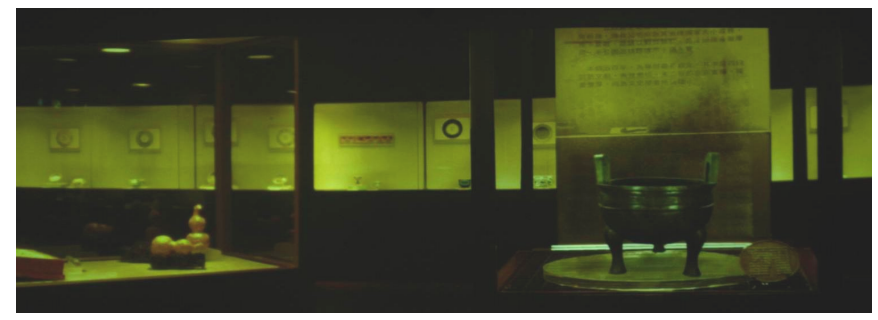

(b) with geometric and photometric calibration

Fig. 2. Virtual Museum Projected from the Fixed Projector System

\subsection{Calibration of Steerable Projectors Regarding to the Display Wall and the Environment}

The Flexible Display includes at least one steerable projector to assist in both presentation and interaction. We show the steerable projector prototype in figure 3 . For the presentation, the steerable projectors act on sub-region enhancement, and information augmentation. For the interaction, they provide stepping user interfaces for the viewers. Both of the two purposes require calibration of the steerable projectors regarding to the display wall or to the environment.

After completing the calibration of the wall-size display system described above, we now have large display coverage on the wall. In case of the steerable projectors overlaying their projections onto the display wall, the projected imageries needs to fine match the display wall. For this purpose, we need to find out the relationship between the steerable projectors and the display wall. Once the relationship is defined, we let the steerable projectors pre-warp the images before the images are projected.

Since the display wall and wall-size display system are calibrated, we can simplify the problem as illustrated in figure 4. Our goal now is to obtain the homographies between the image planes of the steerable projectors and display wall. To minimize the human intervention, we use a pan-tilt-zoom camera to help automate the calibration process. For each steerable projector projecting to a portion of the display wall, we describe four steps required to obtain undistorted projections: First, we let the wall-size display system project a grid pattern and use the camera to capture the pattern for computing the homography $\mathrm{H}_{\mathrm{CD}}$ between the camera view and the wall-size display. We then identify the cross points of the grid patters in the camera view. These cross points are considered as the correspondences between camera view and 


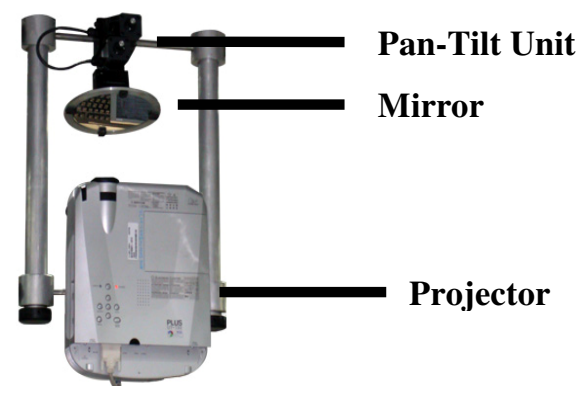

Fig. 3. Steerable Projector (This is a device composed of a projector and a pan-tilt unit mounted a mirror in front of the projector. We can change the projection area by rotating the mirror.)

image plane of the wall-size display system. Notice that at least four correspondences are required to determine the homography. In order to obtain robust results, as many as possible correspondences are used in our implementation. Next, the steerable projector displays another grid pattern. The same process is carried out to find the homography $\mathrm{H}_{\mathrm{CS}}$ between the camera and the steerable projector. Therefore we can derive the homography $\mathrm{H}_{\mathrm{SD}}$ between the steerable projector and the wall-size display system from $\mathrm{H}_{\mathrm{SD}}=\mathrm{H}_{\mathrm{CS}}{ }^{-1} \mathrm{H}_{\mathrm{CD}}$.

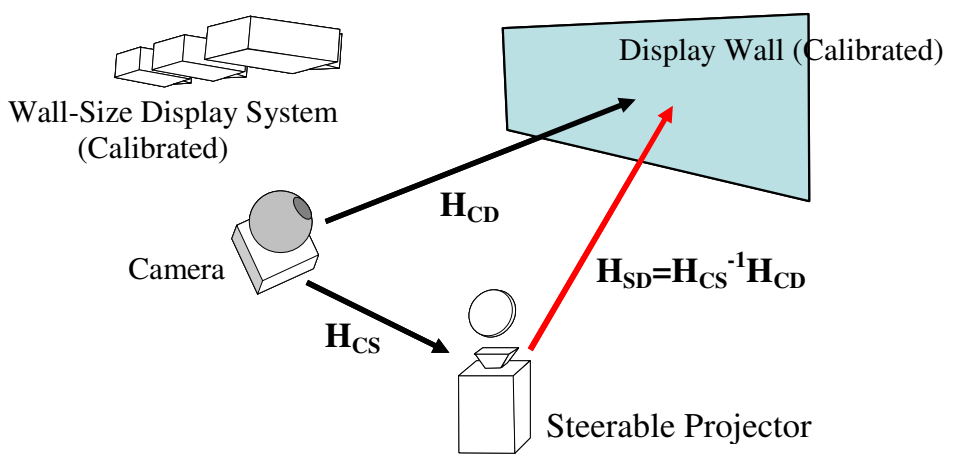

Fig. 4. The transformations among the steerable projector, the camera, and the fixed projector system

Calibration process described above is aimed at a dedicated pose of the mirror said a dedicated position of the projection of the steerable projector. In order that the steerable projection is able to correctly project over the whole display wall, we repeat the process to determine different homographies for different display regions in the calibration stage.

For the steerable projectors projecting onto the environment, the calibration of the steerable projectors regarding to the environment is required in order to deliver undistorted images for the users.

For the steerable projector which is required to project onto the environment, we first move its steerable projection to cover the area, say a target surface of the floor, 
where requires undistorted projection. Here we develop an interactive tool to help the users performing calibration. First, a rectangle pattern is displayed by the steerable projector. Since the steerable projector is not calibrated with regard to the target surface, the projected pattern appears distorted, may be any quadrangle. Next, through the tool, the user iteratively drags the corners of the quadrangle to reshape it as a rectangle. The homography between image plane of the steerable projector and the target surface is then determined, according to the transformation between the origin pattern and the modified pattern.

\subsection{Interactions of the Flexible Display}

We provide the users two kinds of interaction mentioned in system functions section. The first interaction is stepping user interface. While the steerable projector brings widgets around the user, a pan-tilt-zoom camera mounted on upper-side of the display wall then starts monitoring whether the user is stepping on the widget, thus to launch reactions to the display wall. The detection algorithm is mainly differencing operations on image sequences followed by morphological operations to identify a moving object over the widget.

Another interaction, the user accesses the display through the handhelds. We use the PDA, iPAQ hx4700, in the current implementation. During visiting in the environment, the PDA constantly searches nearby Bluetooth access points offered by the display wall. Once the connection built, the PDA offers two modes to interact with the display wall. The first mode, the PDA is a program selector of the display wall. The PDA receives the content program from the display wall and shows it on the screen, the user can drag and throw a certain object to the display wall. The second mode, the PDA can be a touch pad for moving steerable projection over the display wall. The user drags by a stylus on the PDA to freely move the projection, facilitating the high-resolution quality provided by it.

\section{Experimental Results}

In the work of [9], we presented a stereoscopic kiosk for virtual museum, which combines techniques of stereoscopic displays, image-based rendering, virtual reality, and augmented reality. The virtual museum contains 3D scenes implemented by using the image-based technique and by using the model-based technique. For the 3D scenes constructed by geometric models, viewers can interactively view the virtual world from arbitrary viewing directions, but for that built as panoramas, viewers can only watch from some specific viewpoints. In general, the exhibition space implemented by the image-based approach appears to be more realistic. The artifacts are presented as object movies in the virtual exhibition - the image of the artifact shown on screen is selected according to the viewing direction of the user. In this way, artifacts can be rotated and moved in 3D.

In the implementation, we redistribute the virtual museum to the Flexible Display in our laboratory. Flexible Display not only gives its viewers wall-size imagery 
perception that a normal kiosk can not do, but richer interactions that the viewers can better experience with the virtual artifacts. Figure 5 shows a shot of the wall-size display system and the steerable projector in our laboratory. The wall-size display system composing of three projectors projects large scale display coverage on the wall. The steerable projection, at the same time, highlights the artifact placed in the center of the display wall.

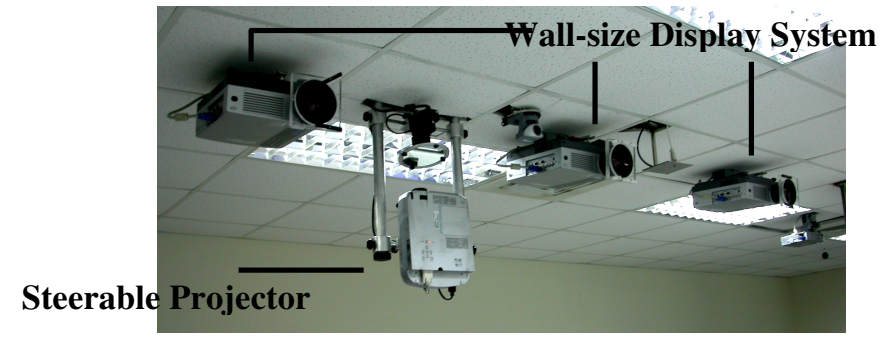

Fig. 5. The Experimental Devices

Figure 6(a) shows the five icons, projected by the steerable projector, around the viewer's foot. Each icon stands for an artifact in this scene. The viewer steps on one of them, the display wall then present the corresponding one on the wall. The viewer steps again to perceive a enlarge mode of the same artifact, as shown in figure 6(b).

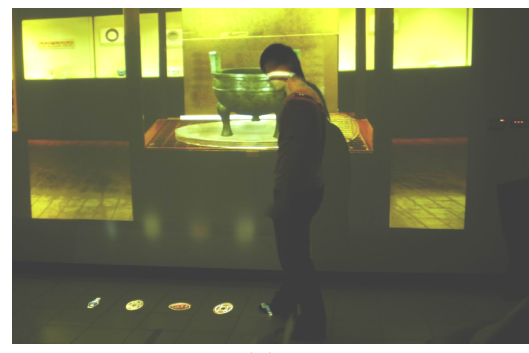

(a)

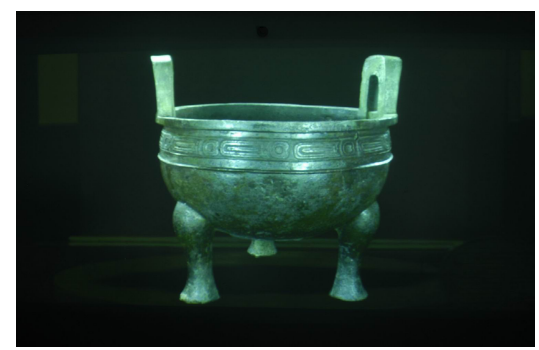

(b)

Fig. 6. The viewer Steps on "Stepping User Interface" to perceive an artifact on the display wall

While in the enlarge mode, the viewer sees two icons standing for instructions "Return" and "Information" projected around the viewer's foot. The steerable projector will augment information over the artifact if the viewer steps on the information icon. Figure 7 shows the full description of the artifact augmented over the display.

To enhance portions of the artifact, the viewer uses the PDA to move the steerable projection. Figure 8 shows the enhanced area presents more details to its viewers. The words in the steerable projection area are clearer the those outside the area. 


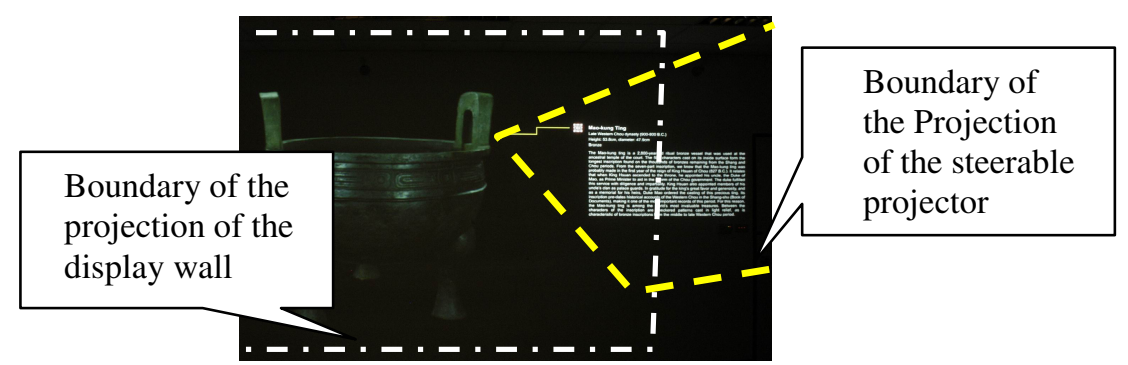

Fig. 7. Extending the Display (The steerable projector extends the display by augmenting the description of the artifact partially outside the display wall. The dash lines on the figure indicate the projection boundaries of the fixed projector system and the steerable projector).
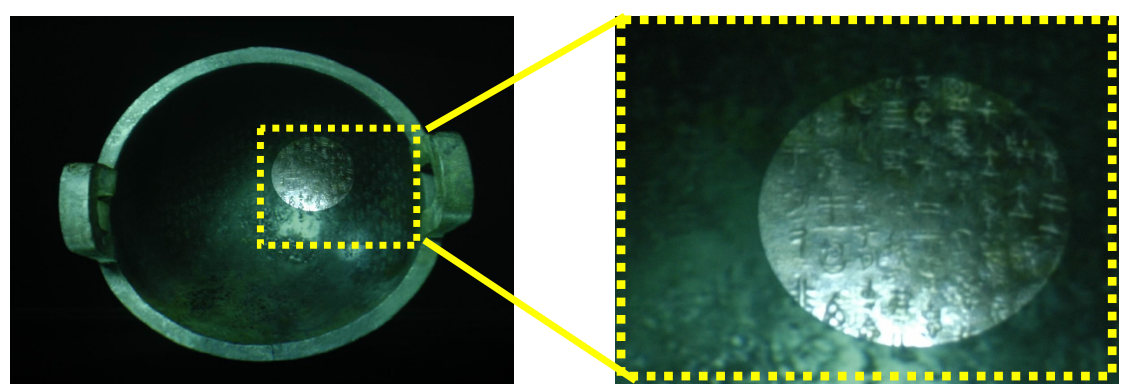

Fig. 8. Enhancing the Display (a)The steerable projection enhances a part of the artifact on the display wall. (b) The zoom-in view shows that the area enhanced by steerable projection are clearer than those outside the enhanced area.

\section{Conclusion}

In this paper, we are investigating how a wall-size display wall and one steerable projector coexisted in the environment can gain benefits from each other. We have developed Flexible Display composing of a wall-size display system, a steerable projector, a camera and PDAs, as well as its three basic functions: sub-region enhancement, information augmentation and stepping user interface. A virtual museum applying Flexible Display is carried out in our laboratory to demonstrate the better capabilities on both the presentation and interaction.

\section{Acknowledgement}

This work was supported in part by the grants of NSC 94-2422-H-002-019 and NSC 94-2752-E-002-007-PAE.

\section{References}

1. R. Raskar, M. S. Brown, R. Yang, W.-C. Chen, G. Welch, H. Towles, B. Seales, and H. Fuch.: Multi-Projector Displays Using Camera-Based Registration. Proc. of IEEE Visualization, 1999, pp. 161-68. 
2. R. Sukthankar, R. G. Stockton, and M. D. Mullin.: Smarter Presentations: Exploiting Homography in Camera-Projector Systems. Proc. of ICCV 2001, 2001.

3. H. Chen, R. Sukthankar, G. Wallace, T.-J. Cham.: Calibrating Scalable Multi-Projector Displays Using Camera Homography Trees. CVPR Technical Sketch, 2001.

4. R. Raskar, J. V. Baar, and J. X. Chai.: A Low-Cost Project Mosaic with Fast Registration. Proc. of Asian Conference on Computer Vision (ACCV2002), 2002.

5. R. Raskar, J. V. Baar, P. Beardsley, T. Willwacher, S. Rao, and C. Forlines.: iLamps: Geometrically Aware and Self-Configuring Projectors. ACM Transactions on Graphics, Vol.22 No.3, p.809-818, July 2003.

6. C. Pinhanez, R. Kjeldsen, A. Levas, G. Pingali, M. Podlaseck, N. Sukaviriya.: Applications of Steerable Projector-Camera Systems. IEEE Workshop on Projector-Camera Systems (Procams 2003), 2003.

7. G. Pingali, C. Pinhanez, A. Levas, R. Kjeldsen, M. Podlaseck, H. Chen, N. Sukaviriya.: Steerable Interfaces for Pervasive Computing Spaces. IEEE Conference on Pervasive Computing and Communications (Percom 2003), pp. 315.

8. Y. P. Tsai, Y. N. Wu, and Y. P. Hung.: Generating a Multiresolution Display by Integrating Multiple Projectors. IEEE Workshop on Projector-Camera Systems (Procams 2003), 2003.

9. W. Y. Lo, Y. P. Tsai, C. W. Chen, Y. P. Hung.: Stereoscopic Kiosk for Virtual Museum. Proceedings International Computer Symposium (ICS 2004), December 2004.

10. A. Majumder, R. Stevens.: Perceptual photometric seamlessness in projector-based tiled displays. ACM Transactions on Graphics, Vol.24 No.1, p.118-139, January 2005

11. M. Brown, A. Majumder, and R. Yang.: Camera-Based Calibration Techniques for Seamless Multiprojector Displays. IEEE Transactions on Visualization and Computer Graphics, Vol. 11, No. 2, March-April, 2005.

12. Y. P. Tsai, S. C. Liao, Y. P. Hung and Z. C. Shih.: Two-Phase photometric calibration for multi-projector displays. Third Taiwanese-French Conference on Information Technology, 2006. 